\title{
Associação entre doença periodontal e aterosclerose subclínica: uma revisão sistemática
}

\author{
Association betwenn periodontal disease and subclinic atherosclerosis: \\ a sistematic review
}

Rafaela das Mercês Batista', Eliana Zandonade², Leonard Hermann Roelke, Adauto Oliveira Emmerich4, Elizabeth Pimentel Rosetti ${ }^{5}$, Maria Del Carmen Bisi Molina ${ }^{6}$, Edson Theodoro dos Santos Neto ${ }^{7}$

\begin{abstract}
Resumo
A associação entre doença periodontal e o desenvolvimento de aterosclerose tem sido pesquisada. Esta revisão sistemática da literatura se propõe a verificar a associação entre a doença periodontal e a aterosclerose subclínica. A pesquisa utilizou as bases de dados: PubMed, Scopus, LILACS, BBO, Biblioteca Cochrane e Scielo com os termos: "doença periodontal", "periodontite", "carótida" e "aterosclerose". Foram excluídos artigos: que apresentaram resumos escritos em idiomas diferentes do português, inglês e espanhol; com abordagem laboratorial em modelos experimentais; sem descrição ou referência a estimadores de associação entre doença periodontal e aterosclerose subclínica; pesquisas que realizaram a medida da doença periodontal por parâmetros diferentes de exames clínicos; pesquisas que não analisaram a espessura da íntima média da artéria carótida através de exame de ultrassom; e que abordassem grupos específicos. Foram comparados os desenhos de estudo, protocolos de medida e os dados relatados. De um total de 63 referências, apenas 10 artigos contemplavam todos os critérios de inclusão, distribuídos em: 1 estudo longitudinal, 6 transversais e 3 casos controles. Observou-se uma heterogeneidade entre os estudos em relação ao método de mensuração da doença periodontal e os desfechos da aterosclerose. Concluiu-se que infecções periodontais são fortemente associadas ao desenvolvimento da aterosclerose subclínica, entretanto os mecanismos envolvidos no processo patogênico ainda permanecem desconhecidos.
\end{abstract}

Palavras-chave: aterosclerose; periodontia; doenças das artérias carótidas.

\begin{abstract}
The association between periodontal disease and the development of atherosclerosis has been studied. The systematic review of literature aims to evaluate the association between periodontal disease and subclinical atherosclerosis. A literature search of the PubMed, Scopus, LILACS, BBO, Cochrane Library and Scielo bibliographic databases was conducted using the following descriptors: "periodontal disease", "periodontitis", "carotid", and "therosclerosis". Articles were excluded if they: presented abstracts written in languages other than Portuguese, English and Spanish; experimental studies; did not contain data testing the degree of association between periodontal disease and subclinical atherosclerosis; did measure the extent of periodontal disease by parameters other than the clinical examination; did not measure the carotid artery intima-media wall thickness by ultrasonography; and covered specific population groups. The studies design, measurement protocols and reported data were compared. A total of 63 papers identified only 10 studies that met the inclusion criteria: 1 longitudinal study, 6 cross-sectional, and 3 case control studies. There was a wide heterogeneity between the studies regarding the methods of measuring periodontal disease and atherosclerosis outcomes. The conclusion is that periodontal infections are strongly associated with the development of subclinical atherosclerosis, however the mechanisms involved on the pathogenic process remain unknown.
\end{abstract}

Keywords: atherosclerosis; periodontics; carotid artery diseases.

Trabalho realizado no Centro de Ciências da Saúde da Universidade Federal do Espírito Santo (UFES) - Vitória (ES), Brasil.

'Especialista em Periodontia; mestre em Saúde Coletiva pelo Programa de Pós-Graduação em Saúde Coletiva do Centro de Ciências da UFES - Vitória (ES), Brasil.

${ }^{2}$ Doutora em Estatística; professora do Programa de Pós-Graduação em Saúde Coletiva do Centro de Ciências da UFES - Vitória (ES), Brasil.

${ }^{3}$ Especialista em Angiologia e Cirurgia Vascular Periférica; Professor auxiliar do Departamento de Clínica Cirúrgica III da UFES - Vitória (ES), Brasil;

«Pós-Doutor em Saúde Pública pela Fundação Oswaldo Cruz - Rio de Janeiro (RJ), Brasil; Professor do Programa de Pós-Graduação em Saúde Coletiva do Centro de Ciências da UFES - Vitória (ES), Brasil.

${ }^{5}$ Doutora em Periodontia; Professora do Centro Universitário de Vila Velha - Vila Velha (ES), Brasil.

'Pós-Doutora em Saúde Coletiva pela Universidade Estadual do Rio de Janeiro (UERJ) - Rio de Janeiro (RJ), Brasil; Professora do Programa de Pós-Graduação em Saúde Coletiva do Centro de Ciências da UFES - Vitória (ES), Brasil.

'Doutorando em Epidemiologia em Saúde Pública na Fundação Oswaldo Cruz - Rio de Janeiro (RJ), Brasil; Professor do Departamento de Medicina Social do Centro de Ciências da UFES -

Vitória (ES), Brasil.

Conflito de interesses: nada a declarar.

Submetido em: 15.09.10. Aceito em: 13.06.11.

J Vasc Bras. 2011;10(3):229-238 


\section{Introdução}

A doença periodontal (DP) é uma doença imunológica crônica multifatorial que se estabelece em resposta a antígenos periodontopatogênicos ${ }^{1}$. Nesta doença, ocorre uma inflamação dos tecidos de suporte dos dentes e, por conseguinte, uma extensão desta inflamação da gengiva para o osso adjacente e ligamento periodontal ${ }^{2}$. A DP é uma doença comum, com a forma leve a moderada afetando de 30 a $50 \%$ da população adulta e a forma grave generalizada afetando de 5 a $15 \%$ dos adultos nos Estados Unidos? ${ }^{3}$. Estes resultados são confirmados por estudos realizados na população brasileira $^{4-5}$. Um estudo ${ }^{6}$ realizado em São Paulo encontrou que $45 \%$ dos indivíduos apresentaram algum comprometimento periodontal e 5\% apresentaram DP grave.

A associação entre DP e doença cardiovascular aterosclerótica tem recebido considerável atençãa ${ }^{7-13}$. Os achados destes estudos, entretanto, têm variado muito ${ }^{14-17}$. Alguns estudos relatam que a infecção periodontal, por meio de um processo inflamatório, pode causar infecção do endotélio vascular ${ }^{18,19}$ e contribuir para a ocorrência da aterosclerose, o que aumenta o risco de isquemia do miocárdio e infarto, sempre precedidos de eventos tromboembolíticos ${ }^{20,21}$. Além desta hipótese, existem várias outras possibilidades sugeridas em que os agentes infecciosos podem induzir ou acelerar a aterosclerose tais como: favorecer o aumento local dos linfócitos, macrófagos, produção de fatores de crescimento teciduais ${ }^{22}$; liberação local de endotoxina (lipopolissacarídeos) e mimetismo molecular da proteína 60 microbiana com a humana induzindo uma reação auto-imune. Além disso, o aumento sistêmico de citocinas com ativação de marcadores inflamatórios e estimulação de pró-coagulantes, pode acarretar trombose e isquemia aguda, além de indução de mudanças nas lipoproteínas resultando em condições préateroscleróticas ${ }^{23}$.

Algumas publicações ${ }^{24-27}$ estabelecem protocolos de pesquisas que visam estudar a possível associação da DP e aterosclerose, utilizando a medida da espessura do complexo intimal da carótida (IMT) como medida da aterosclerose subclínica ${ }^{28}$. Seu uso resulta da possibilidade de predição de desfechos finais de futuros eventos clínicos cardiovasculares a partir de uma técnica não invasiva, já que é realizado usando ultrassom, que fornece um ganho para o diagnóstico, a partir da importância da IMT como fator de risco para a doença cardiovascular (DCV $)^{29-31}$.

Com a progressão da doença aterosclerótica frequentemente ocorre um espessamento visível da IMT ${ }^{32,33}$, bem como é possível observar a presença de placas fibrosas ou calcificadas ${ }^{34,35}$. Medir a espessura das camadas íntima e média da parede da artéria carótida é uma maneira de avaliar um dos mais importantes indicadores da saúde cardiovascular dos pacientes ${ }^{36,37}$.

Diante de tais considerações, esta revisão sistemática da literatura se propõe a investigar a associação entre DP e aterosclerose subclínica, já que a partir dos resultados destes estudos, até mesmo uma associação de magnitude modesta, tem um largo impacto dentro de um contexto de saúde pública, pois o custo para a sociedade diretamente atribuível a sequelas da aterosclerose é grande e a DP é tratável e pode ser prevenida.

\section{Métodos}

\section{Estratégia de busca}

Foi realizada busca de artigos publicados que avaliaram a associação entre DP e aterosclerose pesquisadas nas bases de dados: Scopus, PubMed, LILACS, BBO, Scielo E Biblioteca Cochrane utilizando os descritores "periodontite", "carótida", "aterosclerose" e "doença periodontal", e seus correspondentes em inglês: "periodontitis", "carotid", "atherosclerosis" e "peridontal disease". Os artigos foram buscados em todos os períodos vigentes em cada base de dados até maio de 2010. Também foi utilizada a busca manual nas referências dos artigos e revisões, editoriais e cartas que discutiam esta possível associação. Os artigos encontrados na busca foram lidos por um autor previamente treinado.

Na primeira fase, excluíram-se os trabalhos que se repetiam entre as bases de dados onde foram feitas as buscas ou que foram escritos em idiomas diferentes do português, inglês e espanhol. Além disso, os artigos nos quais os resumos descreviam que não foi testada a associação entre DP e aterosclerose subclínica também foram desconsiderados.

A maioria dos artigos selecionados estava disponível no Portal CAPES. Os artigos indisponíveis on-line foram acessados através da Rede de Comutação Bibliográfica para recuperação de artigos.

\section{Estratégia de análise e seleção}

\section{Critérios de inclusão e exclusão}

Para serem incluídos na análise, os artigos deveriam testar a associação direta entre DP e aterosclerose subclínica por parâmetro estatístico. A informação sobre diagnóstico de aterosclerose deveria ser adquirida através da medida da espessura da íntima média da artéria carótida, por meio de ultrassonografia. Tais pesquisas também não deveriam ser realizadas em modelos experimentais. 
Além disso, as exclusões de pesquisas ocorreram quando estas realizaram a medida da DP por parâmetros diferentes de exames clínicos. Tais estudos não deveriam abordar grupos específicos, por exemplo, grupos portadores de doenças sistêmicas, como transplantados renais, diabéticos ou renais crônicos em hemodiálise.

\section{Resultados}

Na base de dados Scopus, utilizando-se as palavraschave "atherosclerosis", "carotid" e "periodontal disease", foram encontradas 45 referências. Em adição, na mesma base de dados, utilizando-se as palavras-chave: "atherosclerosis", "carotid" e "periodontitis" foram encontradas 36 referências, mas apenas 4 não haviam sido selecionadas na mesma base. Na base de dados PubMed, foram encontradas sete referências que não se repetiam em outras bases. Foram localizadas na base de dados LILACS, duas referências, uma na $\mathrm{BBO}$, e quatro na Biblioteca Cochrane.
Na base de dados Scielo, não foi encontrada nenhuma referência. Todas as bases foram pesquisadas com todos os descritores em português e em inglês. No total, foram analisadas 63 referências.

Quanto aos critérios de inclusão, apenas três artigos estavam em língua diferente do português, inglês e espanhol, e seis não eram artigos de associação entre DP e aterosclerose. Quatro referências não contemplavam resumo, por isso foram lidos na íntegra. Portanto, restaram 54 referências que permaneceram dentro dos critérios de busca de resumos para serem lidos na íntegra (Figura1).

Quanto aos critérios de inclusão dos artigos, o estudo ${ }^{38}$ que avaliou a frequência de ateromas através de radiografias panorâmicas, assim como o trabalho que não avaliou a aterosclerose subclínica pela ultrassonografia ${ }^{39}$, foram excluídos da análise.

As publicações ${ }^{8-10,16,17,23,32,42-53}$ que não testaram a associação por parâmetro estatístico entre as variáveis propostas neste trabalho, DP e aterosclerose, foram excluídas. Já

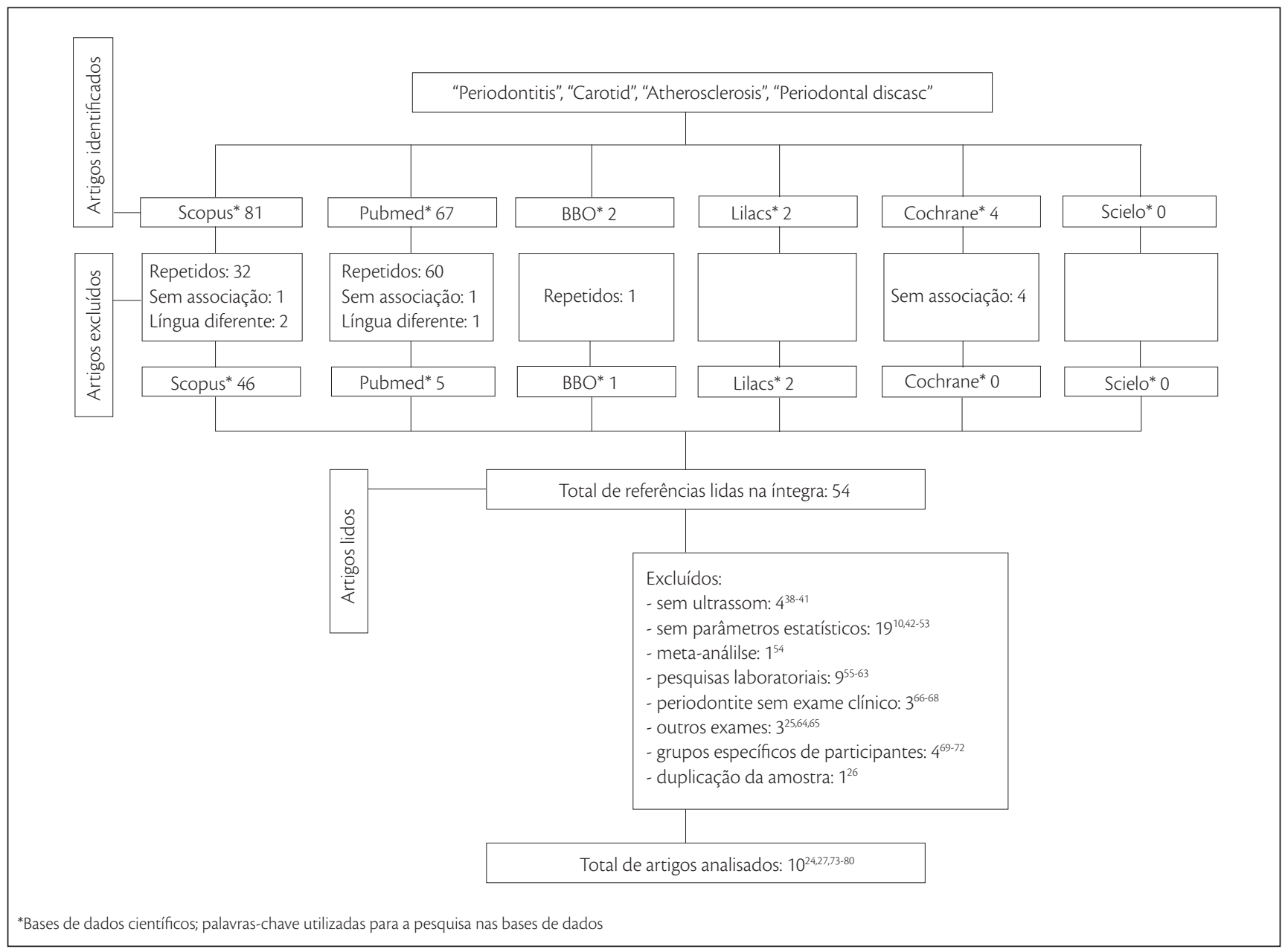

Figura 1. Fluxograma dos artigos selecionados para análise. 
a metanálise $\mathrm{e}^{54}$ foi excluída porque os trabalhos analisados neste estudo fazem parte desta revisão sistemática.

Nove trabalhos ${ }^{55-63}$ foram excluídos por serem pesquisas laboratoriais que não realizaram a ultrassonografia da carótida para avaliar aterosclerose.

Outros três estudos ${ }^{25,64,65}$ que analisaram a DP por parâmetros radiográficos, por meio de radiografias interproximais ou radiografia panorâmica, também foram excluídos.

As pesquisas que mediram a DP por parâmetros diferentes de exames clínicos ou radiográficos ${ }^{66-68}$ foram excluídas, assim como, trabalharam com grupos específicos de pacientes como diabéticos ${ }^{69}$, pacientes em hemodiálise ${ }^{70}$, transplantados renais ${ }^{71}$ e com hipercolesterolemia ${ }^{72}$.

$\mathrm{O}$ estudo ${ }^{26}$ que fazia menção à mesma amostra de outro estudo $^{73}$, também foi excluído.

Portanto, após análise, dez publicações foram selecionadas para integrar a revisão: um estudo longitudinal ${ }^{27}$, seis estudos transversais ${ }^{24,74-78}$ e três estudos do tipo casocontrole $\mathrm{e}^{73,79,80}$, como podem ser observados na Tabela 1 , a qual apresenta a descrição sumária da metodologia dos artigos selecionados. A maior parte dos estudos foi realizada em países industrializados e desenvolvidos, como Estados Unidos (EUA), Suécia entre outros europeus.
O único estudo longitudinal ${ }^{27}$ apresentou um tempo de acompanhamento de seis a nove meses. No que se refere ao tamanho amostral, a maior amostra foi de 6.017 indivíduos $^{24}$ e a menor de 35 indivíduos ${ }^{77}$.

Quanto à coleta de dados periodontais, a medida realizada por meio da profundidade de sondagem de bolsa (PS) e nível de inserção clínica (NI) foi a estratégia mais utilizada para classificar os indivíduos com relação à DP. Entretanto, outros sinais clínicos também foram analisados, tais como: sangramento à sondagem, índice de placa e de mobilidade. Apenas dois trabalhos ${ }^{73,76}$ utilizaram a análise radiográfica como meio complementar ao exame clínico.

A maioria dos estudos classificou os indivíduos de acordo com o grau de severidade periodontal, baseada na perda óssea que ocorreu durante toda a vida do mesmo, realizando a mensuração em seis sítios por dente. Além disso, observouse que a variável de interesse, a DP, não teve uma padronização quanto à descrição dos métodos de coleta dos dados periodontais. É importante mencionar que, para classificação dos indivíduos quanto à variável condição bucal, somente um estudo $^{27}$ avaliou as demais condições dentárias como dentes cariados, perdidos e obturados, através do índice CPO-D.

Em relação à medida da artéria carótida, a artéria carótida comum foi a de escolha da maioria dos estudos para coleta da

Tabela 1. Categorização dos estudos de acordo com a metodologia.

\begin{tabular}{|c|c|c|c|c|c|c|c|}
\hline Publicação & $\begin{array}{l}\text { Desenho } \\
\text { do estudo }\end{array}$ & $\begin{array}{l}\text { Amostra } \\
(\mathrm{n})\end{array}$ & Faixa etária (anos) & Local & $\begin{array}{l}\text { Duração da } \\
\text { pesquisa }\end{array}$ & Coleta de dados periodontais & Coleta da IMT \\
\hline Beck et al. ${ }^{24}$ & Transversal & 6.017 & $52-75$ & EUA & $1996-1998$ & PS, NI, em 6 locais por dente & CCA, ICA, BCA \\
\hline Desvarieux et al. ${ }^{74}$ & Transversal & 711 & 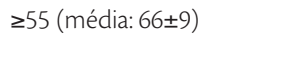 & EUA & não especificou & $\begin{array}{l}\text { PS, NI, em } 6 \text { locais por dente } \\
\text { presença de placa, mobilidade }\end{array}$ & CCA, ICA \\
\hline Ravon et al. ${ }^{76}$ & Transversal & 83 & $\begin{array}{l}\text { US positivos: } \\
\text { homens: média } 69,3 \pm 6,7 \\
\text { mulheres: média } 68,3 \pm 8,3\end{array}$ & EUA & não especificou & $\begin{array}{l}\text { PS, NI, em } 6 \text { locais por dente } \\
\text { sangramento a sondagem, } \\
\text { perda óssea na radiografia }\end{array}$ & ICA \\
\hline Desvarieux et al. $^{75}$ & Transversal & 1.710 & $45-75$ & Alemanha & 1997-2001 & $\begin{array}{l}\text { PS, NI, em } 4 \text { locais por dente } \\
\text { boca dividida- sujeitos alternados }\end{array}$ & $\begin{array}{l}\text { CCA, ICA, } \\
B C A, E C A\end{array}$ \\
\hline Schillinger et al. ${ }^{27}$ & Longitudinal & 411 & $62-76$ & Áustria & 6 a 9 meses & $\begin{array}{l}\text { CPO-D, SLI } \\
\text { CPITN, edêntulos }\end{array}$ & CCA, ICA \\
\hline Soder et al. ${ }^{79}$ & Caso-controle & $\begin{array}{l}67 ; 46 \\
\text { casos }\end{array}$ & $30-40$ & Suécia & não especificou & $\begin{array}{l}\mathrm{PS}, \mathrm{NI} \text {, em } 6 \text { locais por dente } \\
\text { índice gengival, } \\
\text { índice de placa }\end{array}$ & CCA \\
\hline Back et al. ${ }^{77}$ & Transversal & 35 & $\begin{array}{l}52,9 \pm 2,7 \text { sem DPS } \\
54,7 \pm 3,2 \text { com DP }\end{array}$ & Suécia & $\begin{array}{l}1985-2001 / \\
2003\end{array}$ & $\begin{array}{l}\text { PS, NI, em } 6 \text { locais por dente } \\
\text { sangramento a sondagem, } \\
\text { Índice de placa }\end{array}$ & CCA \\
\hline Cairo et al. $2008^{80}$ & Caso-controle & $90 ; 45$ casos & $18-40$ & Itália & Não especificou & $\begin{array}{l}\text { PS, NI, em } 6 \text { locais por dente } \\
\text { presença de placa, sangramento a } \\
\text { sondagem }\end{array}$ & CCA \\
\hline Demmer et al. ${ }^{78}$ & Transversal & 1.745 & $\geq 45$ & Pomerânia & $1997-2001$ & $\begin{array}{l}\text { PS, NI, em } 4 \text { locais por dente } \\
\text { Lados D e E alternados, sangramento } \\
\text { a sondagem no IC, C, } 1^{\circ} \mathrm{M}\end{array}$ & CCA \\
\hline Soder et al. ${ }^{73}$ & Caso-controle & $\begin{array}{l}111 ; 80 \\
\text { casos }\end{array}$ & $\begin{array}{l}54,4 \pm 3,0 \text { com DP } \\
53,2 \pm .2,8 \text { sem DP }\end{array}$ & Suécia & $1985-2003$ & $\begin{array}{l}\text { PS, NI, sangramento a sondagem em } 6 \\
\text { locais por dente, índice de placa, } 14 \text { rx }\end{array}$ & CCA \\
\hline
\end{tabular}

CCA - artéria carótida comum; ICA - artéria carótida interna; ECA- artéria carótida externa; BCA- bifurcação da artéria carótida; CPITN- índice de necessidade de tratamento periodontal; CPO-D- índice de dentes cariados, perdidos e obturados; DP- doença periodontal; IMT- espessura do complexo intimal da carótida; OR- odds ratio; PS - profundidade de sondagem; NI- nível de inserção; SLI- índice de Silness-Loe; lado D- lado direito; lado E- lado esquerdo; US- ultrassonografia; IC- incisivo; C- canino; $1^{\circ} \mathrm{M}$ - primeiro molar; rX- radiografia 
medida da espessura do complexo intimal da artéria carótida (IMT) e placas ateroscleróticas nas ultrassonografias (US), exceto em um estudo ${ }^{76}$. Além da artéria carótida comum (CCA), outra análise realizada foi a média das medidas das artérias carótida externa (ECA), interna (ICA) e bifurcação (BCA).
A Tabela 2 apresenta os resultados dos estudos. Quanto à variável medida da aterosclerose subclínica, observou-se que não há um padrão estabelecido para a categorização, pois foram determinadas tanto qualitativas nominais dicotômicas, quanto quantitativas ordinais ou nominais com

Tabela 2. Categorização dos estudos segundo os resultados da associação entre periodontite e aterosclerose subclínica.

\begin{tabular}{|c|c|c|c|c|c|c|}
\hline Publicação & Desfecho & Variável de interesse & $\begin{array}{l}\text { Análise } \\
\text { estatística }\end{array}$ & Valor $\mathrm{p}$ & Estimador de risco & $\begin{array}{l}\text { Intervalo de confiança } \\
(95 \%) \text { após ajuste }\end{array}$ \\
\hline $\begin{array}{l}\text { Beck } \\
\text { et al. }{ }^{24}\end{array}$ & $\mathrm{IMT} \geq 1 \mathrm{~mm}$ & $\begin{array}{l}\text { DP: perda óssea } \geq 3 \mathrm{~mm} \\
\text { leve }<10 \% \\
\text { moderada } 10-30 \% \text {, } \\
\text { grave }>30 \%\end{array}$ & $\begin{array}{l}\text { Regressão } \\
\text { logística } \\
\text { múltipla }\end{array}$ & $p<0,01$ & $\begin{array}{l}\text { OR: } 1,31 \text { DP grave } \\
\text { OR: } 1,10 \text { DP moderada }\end{array}$ & $\begin{array}{l}1,03-1,66 \\
0,89-1,35\end{array}$ \\
\hline $\begin{array}{l}\text { Desvarieux } \\
\text { et al. } .^{74}\end{array}$ & $\begin{array}{l}\text { Presença de Placa } \\
\text { carotídea }\end{array}$ & $\begin{array}{l}\text { Perda dentária: } 0 \text { a } 9 \text { dentes } \\
\text { ausentes } \\
10 \text { a 19: dentes ausentes } \\
20 \text { a 31: dentes ausentes } \\
\text { edêntulos: DP grave para PS } \geq 5 \mathrm{~mm} \\
\text { e NI } \geq 4 \mathrm{~mm}\end{array}$ & $\begin{array}{l}\text { Regressão linear } \\
\text { logística }\end{array}$ & $p<0,05$ & $\begin{array}{l}\text { OR: 1,95 (para } 10 \text { a } 19 \\
\text { dentes faltando) }\end{array}$ & $1,25-3,04$ \\
\hline $\begin{array}{l}\text { Ravon } \\
\text { et al. }\end{array}$ & $\begin{array}{l}\text { Estenose de carótida } \\
\text { maior } \\
\text { do que } 80 \% \text { de } \\
\text { diâmetro }\end{array}$ & $\begin{array}{l}\text { DP: distância CEJ-BL } \geq 4 \mathrm{~mm} \\
\text { em } \geq 30 \% \text { dos dentes }\end{array}$ & $\begin{array}{l}\text { Correlações de } \\
\text { Spearman }\end{array}$ & $\begin{array}{l}p<0,05 \\
p<0,001\end{array}$ & $\begin{array}{l}C A L \geq 5,0 \mathrm{~mm}, r=0,23 \\
C E J-B L \geq 4,0 \mathrm{~mm}, r=0,46\end{array}$ & - \\
\hline $\begin{array}{l}\text { Desvarieux } \\
\text { et al. } .^{75}\end{array}$ & $\begin{array}{l}\text { IMT média e } \\
\text { Prevalência de placa } \\
\text { carotídea } \geq 1 \mathrm{~mm}\end{array}$ & $\begin{array}{l}\text { Perda dentária: } 0 \text { a } 8 \text { dentes } \\
\text { ausentes } \\
9 \text { a } 15 \text { dentes ausentes } \\
16 \text { a } 31 \text { dentes ausentes } \\
\text { edêntulos } \\
\text { DP grave para } P S \geq 5 \mathrm{~mm} \text { e } \mathrm{NI} \geq 4 \\
\mathrm{~mm}\end{array}$ & $\begin{array}{l}\text { Regressão linear } \\
\text { logística }\end{array}$ & $p<0,05$ & $\begin{array}{l}\text { Homens: OR: 1,66 ( } 16 \text { a } \\
31 \text { dentes faltando) }\end{array}$ & $1,04-2,65$ \\
\hline $\begin{array}{l}\text { Schillinger } \\
\text { et al. }{ }^{27}\end{array}$ & $\begin{array}{l}\text { Estenose de carótida } \\
\text { Se aumentou } 1 \\
\text { categoria: } \\
0 \text { a } 29 \%, 30 \text { a } 49 \%, 50 \\
\text { a } 69 \%, 70 \text { a } 89 \%, 90 \text { a } \\
99 \%, 100 \%\end{array}$ & $\begin{array}{l}\text { Bolsas periodontais leve com } 4 \text { ou } \\
5 \text { mm de sondagem, e grave : } \mathrm{NI} \geq 6 \\
\text { mm usando o CPITN } \\
\text { SLI: Índice de placa de } 0 \text { a } 3 \\
\text { CPO-D: dentes cariados, perdidos, } \\
\text { obturados }\end{array}$ & $\begin{array}{l}\text { Regressão } \\
\text { logística } \\
\text { múltipla }\end{array}$ & $\begin{array}{l}\text { 0,046 perdidos } \\
0,032 \text { CPO-D } \\
0,021 \text { SLI } \\
0,16 \text { CPITN }\end{array}$ & $\begin{array}{l}\text { OR: } 2,69 \\
\text { OR: } 1,11 \\
\text { OR: } 1,77 \\
\text { OR: } 1,51\end{array}$ & $\begin{array}{l}1,62-4,17 \\
1,01-1,22 \\
1,09-2,79 \\
0,89-2,45\end{array}$ \\
\hline $\begin{array}{l}\text { Soder } \\
\text { et al. }{ }^{79}\end{array}$ & IMT, cIMA & $\begin{array}{l}\text { DP, placa, inflamação gengival, } \\
\text { severa em mulheres }\end{array}$ & $\begin{array}{l}\text { Regressão } \\
\text { logística } \\
\text { múltipla }\end{array}$ & $\begin{array}{l}0,019 \text { IMT } \\
0,028 \mathrm{cIMA}\end{array}$ & $\begin{array}{l}\text { OR: } 6,05 \\
\text { OR: } 5,41\end{array}$ & $\begin{array}{l}1,34-27,35 \\
1,20-24,43\end{array}$ \\
\hline $\begin{array}{l}\text { Back } \\
\text { et al. }{ }^{77}\end{array}$ & $\begin{array}{l}\text { cIMA, placa } \\
\text { aterosclerótica }\end{array}$ & $\begin{array}{l}\mathrm{DP}=\mid \text { com } \mathrm{NI}>3 \mathrm{~mm} \text {, Sangramento } \\
\text { a sondagem }>20 \text { e } \mathrm{PS}>2.2 \text { (média) }\end{array}$ & $\begin{array}{l}\text { Teste } t \text { de } \\
\text { Student, teste } \\
\text { exato de } \\
\text { Fisher, teste } \\
\text { Ude Mann } \\
\text { Whitney }\end{array}$ & $p<0,05$ & $\begin{array}{l}\mathrm{cIMA}\left(\mathrm{mm}^{2}\right) 11,4 \pm 1.8 \\
\text { sem DP } \\
13,8 \pm 3,3 \text { com DP }\end{array}$ & - \\
\hline $\begin{array}{l}\text { Cairo } \\
\text { et al. }\end{array}$ & $\mathrm{IMT} \geq 0.82 \mathrm{~mm}$ & $\begin{array}{l}\text { DP grave generalizada (pelo } \\
\text { menos } 30 \% \text { dos locais com perda } \\
\text { de inserção }>3 \text { mm e perda óssea } \\
\text { alveolar de }+1 / 3 \text { raiz }\end{array}$ & $\begin{array}{l}\text { Regressão linear } \\
\text { logística }\end{array}$ & $p=0,0002$ & OR: 8,55 & $2,38-39,81$ \\
\hline Demmer et. $^{78}$ & $\begin{array}{l}\text { IMT média de } 10 \\
\text { medidas } \\
\text { consecutivas }\end{array}$ & $\begin{array}{l}\text { Severidade de DP de } 3 \text { a } 10-16 \\
\text { definições dicotômicas de limiar } \\
\text { baseadas em sítios com PS ou } \\
\mathrm{NI} \geq \text { ao limiar de severidade. (os } \\
\text { participantes foram definidos como } \\
\text { sim/não tendo alguma PS } \geq 3 \mathrm{~mm} \text { ) }\end{array}$ & Correlações & $p<0,05$ & $\begin{array}{l}r=0,14 \text { homens } \mathrm{p} / \mathrm{NI} \\
\geq 6 \mathrm{~mm} \\
r=0,13 \text { mulheres } \mathrm{p} / \\
\mathrm{PS} \geq 5 \mathrm{~mm}\end{array}$ & - \\
\hline $\begin{array}{l}\text { Soder } \\
\text { et al. }\end{array}$ & $\begin{array}{l}\text { Aumento de IMT, } \\
\text { cIMA }\end{array}$ & $\begin{array}{l}\text { Periodontite crônica=pelo menos } \\
\text { um sítio com PS } \geq 5 \mathrm{~mm}\end{array}$ & $\begin{array}{l}\text { Regressão } \\
\text { logística } \\
\text { múltipla }\end{array}$ & $p<0,05$ & $\begin{array}{l}\text { OR: } 3,89 \text { IMT } \\
\text { OR: } 5,31 \text { cIMA }\end{array}$ & $\begin{array}{l}1,43-10,60 \text { IMT } \\
1,8-15,68 \text { cIMA }\end{array}$ \\
\hline
\end{tabular}

CPITN- índice de necessidade de tratamento periodontal; CPO-D- índice de dentes cariados, perdidos e obturados; CAL- perda de inserção clínica; CEJ-BL- junção cemento-esmalte; DPdoença periodontal; IMT- espessura da íntima-média; CIMA- análise da área transversal da íntima-média da carótida; OR- odds ratio; PS- profundidade de sondagem; NI- nível de inserção; SLI- índice de Silness-Loe; r- correlação 
várias categorias. Além da medida da IMT, através do aumento de espessura ou presença de placa aterosclerótica ${ }^{74,75-77}$, outra medida utilizada pelos pesquisadores para avaliar o desfecho foi a cIMA ${ }^{73,77,79}$ que consistiu em análise da área transversal da íntima-média da carótida. A cIMA é calculada quando o que se deseja é compensar o efeito do estreitamento da distensão arterial (secundária ao aumento da pressão arterial) na espessura da parede ${ }^{73}$.

Na maioria dos estudos, foi realizada tanto a análise bivariada, para testar a associação entre DP e DCV, quanto a análise multivariada, que permite identificar a influência de diversos fatores de confundimento nesta relação.

$\mathrm{Na}$ Tabela 3, estão apresentados os fatores de confundimento que permaneceram estatisticamente significativos após análise multivariada com valores menores que 5\% nos estudos analisados.

Deve-se ressaltar que apenas um trabalho ${ }^{78}$ não fez ajustes considerando outros fatores de confundimento. Por outro lado, a associação entre DP e DCV permaneceu significante após ajuste, somente para homens com 16 a 31 dentes ausentes no estudo de Desvarieux et al. ${ }^{75}$. Em outro estudo ${ }^{27}$, foi encontrado que ausência de dentes foi uma preditora de estenose progressiva carotídea após análise de regressão logística multivariável, sugerindo que pode ser devido à DP grave provavelmente o maior determinante de perda dentária nestes pacientes.

Outro estudo ${ }^{76}$ destaca-se porque os sujeitos com uma faixa etária média de 69 anos para homens e 68 anos para mulheres apresentaram resultados de exame de ultrassonografia positivo, enquanto os de faixa etária mais baixa, o resultado foi negativo. Apenas esta variável, dentre as demais associações avaliadas como sexo, etnia, fumo, história médica, permaneceu significante após o ajuste. Entretanto, o hábito de fumar e a idade foram as variáveis que mais se apresentaram como fatores predisponentes tanto da DP quanto da DCV (Tabela 3).

\section{Discussão}

Embora a maioria dos estudos ${ }^{24,73-75}$ analisados tenha encontrado uma associação positiva entre DP grave e o desenvolvimento de aterosclerose, as limitações metodológicas causam dúvidas da validade dos desfechos e conclusões. Existe uma variação considerável quanto à qualidade metodológica, incluindo pequeno tamanho de amostra, número limitado de análises estatísticas, controle inadequado para fatores de confundimento, avaliação inadequada das medidas da DP e a dependência de dados transversais. Contudo, a associação entre DP e o desenvolvimento da aterosclerose parece ser plausível.
A maioria das pesquisas foi realizada em países industrializados, fato que pode ser explicado pela necessidade de investigação clínica por meio de profissionais altamente especializados e instrumentos de alta densidade tecnológica para estabelecer a evolução tanto da aterosclerose quanto da DP, o que torna a pesquisa mais onerosa para ser realizada em países em desenvolvimento, com custos elevados que nem sempre estarão disponíveis.

As pesquisas transversais mostram-se importantes porque possibilitam a inclusão de grandes amostras para avaliação de um volume considerável de dados, o que é praticamente impossível de ser realizado em estudos do tipo coorte devido ao alto custo financeiro. Entretanto, tais desenhos são indicados para diagnósticos da situação atual do fenômeno em análise, porém não são adequados para predizer estimativas de risco populacional. Quando se trata de DP, é possível mensurar percentuais de história pregressa da doença através da perda de inserção clínica, mas não é possível estabelecer qual fenômeno antecedeu ao outro: a aterosclerose ou a DP.

Os estudos longitudinais são difíceis de serem realizados para analisar os fatores de interesse, pois tanto a DP quanto a aterosclerose são doenças crônicas de evolução lenta. A partir da medida da espessura da IMT de carótida é possível diagnosticar a aterosclerose como desfecho intermediário subclínico.

Tabela 3. Fatores de confundimento significantes ajustados para aterosclerose $(p<0,05)$.

\begin{tabular}{|c|c|c|c|c|c|c|c|c|c|c|}
\hline \multirow[b]{2}{*}{$\begin{array}{l}\text { Fatores de } \\
\text { confundimento }\end{array}$} & \multicolumn{10}{|c|}{ Artigos } \\
\hline & 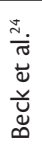 & 苞 & 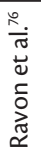 & 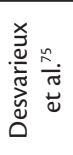 & 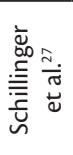 & 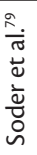 & $\begin{array}{l}\frac{E}{\sigma} \\
\stackrel{\Delta}{0} \\
\stackrel{v}{U} \\
\tilde{D}\end{array}$ & 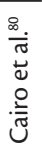 & 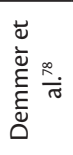 & 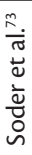 \\
\hline Sexo & $x$ & $x$ & & & $x$ & & & & & $x$ \\
\hline Idade & $x$ & $x$ & $x$ & $x$ & $x$ & $x$ & & $x$ & & $x$ \\
\hline Educação & & $x$ & & $x$ & & $x$ & & $x$ & & \\
\hline Raça & & $x$ & & & & & & & & \\
\hline Fumo & $x$ & $x$ & & $x$ & $x$ & $x$ & $x$ & & & \\
\hline IMC & & & & $x$ & $x$ & $x$ & & & & $x$ \\
\hline $\begin{array}{l}\text { História de DCV } \\
\text { familiar }\end{array}$ & & & & & $x$ & $x$ & & & & \\
\hline Hipertensão & $x$ & $x$ & & $x$ & $x$ & $x$ & & & & \\
\hline Glicose & & & & & $x$ & & & & & \\
\hline Triglicerídios & $x$ & & & $x$ & & & & & & \\
\hline LDL & $x$ & $x$ & & $x$ & & & & & & \\
\hline $\mathrm{HDL}$ & & $x$ & & $x$ & & & & & & \\
\hline Colesterol & & & & & & $x$ & & & & $x$ \\
\hline Diabetes & $x$ & $x$ & & $x$ & $x$ & $x$ & & & & \\
\hline Taxa cintura quadril & $x$ & & & & & & & & & \\
\hline Hiperlipidemia & & & & & $x$ & & & & & \\
\hline
\end{tabular}

DCV- Doença cardiovascular; HDL- lipoproteína de alta densidade; IMC- índice de massa corporal; LDL- lipoproteína de baixa densidade 
A presente revisão demonstra clara associação entre DP e aterosclerose subclínica, pois todos os artigos analisados, independentemente do desenho de estudo, mostraram associação estatisticamente significante, mesmo após ajuste por fatores de confundimento.

Uma limitação na comparação dos resultados analisados é a diversidade de análises da variável desfecho, mesmo tendo como critério de inclusão a medida da aterosclerose por meio de ultrassonografia. Este achado condiz com o achado da revisão sistemática que pesquisou a associação entre DP e incidência de doença cardíaca coronária ${ }^{15}$. Outro aspecto diz respeito à diferença nos padrões adotados para medir a espessura da íntima-média de carótida, ao utilizar tanto um valor para IMT $\geq 1 \mathrm{~mm}^{24,75}$, quanto diminuindo este limiar para $\mathrm{IMT} \geq 0,82 \mathrm{~mm}^{80}$.

Outra limitação nas análises dos estudos epidemiológicos da presente revisão é a diversidade de medidas da DP e a falta de consenso na definição e classificação da mesma. Medidas confiáveis da DP deveriam utilizar a profundidade de sondagem de bolsa e nível de perda de inserção clínica em seis locais por dente $e^{24,73,74,76-80}$.

É notável o papel que a idade e o hábito de fumar exercem no desenvolvimento da DP e DCV. Entretanto, permanece inconclusiva a explicação sobre qual mecanismo fisiopatológico levaria ao aumento da aterosclerose $\mathrm{e}^{18,23}$.

Alguns estudos ${ }^{73,76-78,80}$ apresentaram falta de controle de variáveis independentes e desfecho, o que pode diminuir a confiabilidade dos seus resultados e conclusões, bem como as limitações metodológicas podem não permitir adequadas conclusões sobre o possível efeito da DP sobre os desfechos da aterosclerose. Uma possível relação causal permanece desconhecida. Estudos analíticos com maior rigor metodológico, empregando medidas confiáveis para avaliar a exposição e desfecho, serão úteis em pesquisas futuras.

Ainda são poucos os estudos ${ }^{10}$ que avaliaram a resposta do tratamento periodontal na melhora da disfunção endotelial ou diminuição do espessamento da IMT, mas talvez este seja o caminho das pesquisas que investigam esta possível associação. Esta revisão sistemática sugere a necessidade de padronização metodológica dos estudos a serem realizados para possibilitar futuramente a sintetização dos resultados em mais estudos de metanálise ${ }^{80}$. Com base na predominância das publicações encontradas, os estudos subsequentes sobre DP e aterosclerose devem ser preferencialmente de coorte prospectivo, com cálculo prévio do tamanho de amostra e tempo necessário para acompanhamento do desenvolvimento da DP para se testar variáveis relacionadas ao desenvolvimento da aterosclerose.

\section{Conclusões}

Infecções periodontais foram fatores fortemente associadas ao desenvolvimento de aterosclerose subclínica. Entretanto, os mecanismos pelos quais este fator age no desenvolvimento da progressão deste desfecho, ainda não estão esclarecidos. As doenças cardiovasculares e as infecções periodontais são fenômenos complexos. Novos estudos devem apontar na direção de acompanhar o efeito da terapia periodontal na progressão da aterosclerose.

Fatores gerais, relacionados com idade, sexo e hábito de fumar, que interferem no desenvolvimento de doenças cardiovasculares e DP deverão ser controlados nos estudos.

Devido à característica metodológica dos estudos incluídos, novos estudos com maior rigor metodológico e maior número amostral devem ser realizados para avaliar a real associação entre DP e aterosclerose. Além disso, é necessário que estas pesquisas sejam também realizadas em países com características próprias, como nos países em desenvolvimento.

Estudos futuros envolvendo a associação das condições bucais e aterosclerose devem ser padronizados para todos os elementos metodológicos, a fim de permitir avanços no conhecimento científico deste fenômeno.

\section{Referências}

1. Pihlstrom BL, Michalowicz BS, Johnson NW. Periodontal diseases. Lancet. 2005;366(9499):1809-20.

2. Cortelli JR, Lotufo RFM, Oppermann RV, et al. Glossário da Sociedade Brasileira de Periodontologia. Rev Periodontia. 2005;15(4):5-61.

3. Burt B; Research, Science and Therapy Committee of the American Academy of Periodontology. Position paper: epidemiology of periodontal diseases. J Periodontol. 2005;76(8):1406-19.

4. Bassani DG, Silva CM, Oppermann RV. Validity of the Community Periodontal Index of Treatment Need's (CPITN) for population periodontitis screening. Cad Saúde Pública. 2006;22(2):227-83.

5. Susin C, Albandar JM. Aggressive periodontitis in an urban population in southern Brazil. J Periodontol. 2005;76(3):468-75.

6. Cortelli JR, Cortelli SC, Pallos D, et al. Prevalência de periodontite agressiva em adolescentes e adultos jovens do Vale do Paraíba. Pesqui Odontol Bras. 2002;16(2):163-8.

7. Accarini R, Godoy MF. Doença periodontal como fator de risco para síndromes coronarianas agudas. Arq Bras Cardiol. 2006;87(5):592-6.

8. Persson GR, Persson RE. Cardiovascular disease and periodontitis: an update on the associations and risk. J Clin Periodontol. 2008;35 (Suppl 8):362-79.

9. Ridker PM, Silvertown JD. Inflammation, C-reactive protein, and atherothrombosis. J Periodontol. 2008;79 (Suppl 8):1544-51.

10. Piconi S, Trabattoni D, Luraghi C, et al. Treatment of periodontal disease results in improvements in endothelial dysfunction 
and reduction of the carotid intima-media thickness. FASEB J. 2009;23(4):1196-204.

11. Tonetti MS. Periodontitis and risk for atherosclerosis: an update on intervention trials. J Clin Periodontol. 2009;36(Suppl 10):15-9.

12. Beck JD, Couper DJ, Falkner KL, et al. The periodontitis and vascular events (PAVE) pilot study: adverse events. J Periodontol. 2008;79(1):90-6.

13. Rizzo M, Corrado E, Coppola G, et al. Prediction of cardio- and cerebro-vascular events in patients with subclinical carotid atherosclerosis and low HDL-cholesterol. Atherosclerosis. 2008;200(2):389-95.

14. Friedewald VE, Kornman KS, et al. The American Journal of Cardiology and Journal of Periodontology editors' consensus: periodontitis and atherosclerotic cardiovascular disease. Periodontol. 2009;80(7):1021-32.

15. Humphrey LL, Fu R, Buckley DI, et al. Periodontal disease and COronary heart disease incidence: a systematic review and metanaanalysis. J Gen Intern Med. 2008;23(12):2079-86.

16. US Preventive Services Task Force. Using nontradicional risk factors in coronary heart disease risk assessment: US Preventive Services Task Force recommendation statement. Ann Intern Med. 2009;151(7):474-82.

17. Helfand $M$, Buckley DI, Freeman $M$, et al. Emerging risk factors for coronary heart disease: a summary of systematic reviews conducted for the US Preventive services task force. Ann Intern Med. 2009;151(7):496-507.

18. Fong IW. Emerging relations between infections diseases and coronary artery disease and atherosclerosis. CMAJ. 2000;163(1):49-56.

19. Libby P. Inflammation and cardiovascular disease mechanisms. Am J Clin Nutr. 2006;83(2):456S-60S.

20. Herzberg MC, Meyer MW. Dental plaque, platelets, and cardiovascular diseases. Ann Periodontol. 1998;3(1):151-60.

21. Van Dyke TE. Inflammation and periodontal diseases: a reappraisal J. Periodontol. 2008;79 Suppl 8:1501-2.

22. Serrano Junior CV, Souza JA. Doença periodontal como potencial fator de risco para síndromes coronárias agudas. Arq Bras Cardiol. 2006;87(2):562-3.

23. Tzorbatzoglou ID, Sfyroeras GS, Giannoukas AD. Periodontitis and carotid atheroma; is there a causal relationship? Int Angiol. 2010;29(1):27-9.

24. Beck JD, Elter JR, Heiss G, et al. Relationship of periodontal disease to carotid artery intima-media wall thickness: the atherosclerosis risk in communities (ARIC) study. Arterioscler Thromb Vasc Biol. 2001;21(11):1816-22.

25. Leivadaros $E$, van der Velden $U$, Bizzarro $S$, et al. A pilot study into measurements of markers of atherosclerosis in periodontitis. J Periodontol. 2005;76(1):121-8

26. Söder PO, Söder B, Nowak J, et al. Early carotid atherosclerosis in subjects with periodontal diseases. Stroke. 2005;36(6):1195-200.

27. Schillinger T, Kluger W, Exner $M$, et al. Dental and periodontal status and risk for progression of carotid atherosclerosis: the inflammation and carotid artery risk for atherosclerosis study dental substudy. Stroke. 2006;37(9):2271-6

28. van der Meer IM, Bots ML, Holfman A, et al. Predictive value of noninvasive measures of atherosclerosis for incident myocardial infarction: the Rotterdam study.Circulation.2004;109(9): 1089-94.

29. Torres FS, Moreira CM, Vianna FF, et al. Medida da espessura das camadas íntima e média das artérias carótidas para avaliação do risco cardiovascular. Rev Bras Hipertens. 2007;14(3):167-71.

30. O'Leary DH, Polak JF, Kronmal RA, et al. Carotid-artery intima and media thickness as a risk for myocardial infarction and stroke in older adults. Cardiovascular Health Study Collaborative Research Group. N Engl J Med. 1999;340(1):14-22.

31. O'Leary DH, Polak JF. Intima-media thickness: a tool for atherosclerosis imaging and event prediction. Am J Cardiol 2002;90(10C):18L-21L.

32. Jacobs Junior DR, Crow RS. Subclinical cardiovascular disease markers applicable to studies of oral health: multiethnic study of atherosclerosis. Ann N Y Acad Sci. 2007;1098:269-87.

33. Stein JH, Korcarz CE, Hurst RT, et al. Use of carotid ultrasound to identify subclinical vascular disease and evaluate cardiovascular disease risk: a consensus statement from the American Society of Echocardiography Carotid Intima-Media Thickness Task Force. Endorsed by the Society for Vascular Medicine. I Am Soc Echocardiogr. 2008;21(2):93-111.

34. Chambless LE, Folsom AR, Clegg LX, et al. Carotid wall thickness is predictive of incident clinical stroke: the Atherosclerosis Risk in Communities (ARIC) study. Am J Epidemiol. 2000;151(5):478-87.

35. Cao JJ. Arnold AM, Manolio TA, et al. Association of carotid artery intima-media thickness, plaques, and C-reactive protein with future cardiovascular disease and all-cause mortality: the cardiovascular health study. Circulation. 2007;116(1):32-8.

36. Lorenz MW, Markus HS, Bots ML, et al. Prediction of clinical cardiovascular events with carotid intima-media thickness: a systematic review and meta-analysis. Circulation. 2007;115(4):459-67.

37. Lim TK, Lim E, Dwivedi G, et al. Normal value of carotid intima-media thickness - a surrogate marker of atherosclerosis: quantitative assessment by B-mode carotid ultrasound. J Am Soc Echocardiogr. 2008;21(2);112-6.

38. Quiñónéz P, Calderón V, Quintana M. Frecuencia de ateromas em radiografias panorámicas de pacientes mayores de 40 años con enfermedad periodontal atendidos em una clínica dental universitaria. Rev Estomatol Hered. 2006;16(2):110-4.

39. Aquino AR. Detecção de bactérias periodontais cultiváveis e não cultiváveis em placas ateromatosas. [dissertação]. Natal: Universidade Federal do Rio Grande do Norte, 2008.

40. Beckstrom BW, Horsley SH, Scheetz JP, et al. Correlation between carotid area calcifications and periodontitis: a retrospective study of digital panoramic radiographic findings in pretreatment cancer patients. Oral Surg Oral Med Oral Pathol Oral Radiol Endod. 2007;103(3):359-66.

41. Cairo F, Gaeta C, Dorigo W, et al. Periodontal pathogens in atheromatous plaques. A controlled clinical and laboratory trial. J Periodontal Res. 2004;39(6):442-6.

42. Okuda K, Kato T, Ishihara K. Involvement of periodontopathic biofilm in vascular diseases. Oral Dis. 2004;10(1):5-12.

43. Beck JD, Pankow J, Tyroler HA, et al. Dental infections and atherosclerosis. Am Heart J.1999;138(5 Pt 2):S528-33.

44. Beck JD, Offenbacher S, Williams R, et al. Periodontitis: a risk factor for coronary heart disease? Ann Periodontol. 1998;3(1):127-41. 
45. Goldstein LB, adams R, Alberts MJ, et al. Primary prevention of ischemic stroke. A guideline from the American Heart Association/American Stroke Association Stroke Council: Cosponsored by the Atherosclerotic Peripheral Vascular Disease Interdisciplinary Working Group; Cardiovascular Nursing Council; Clinical Cariology Council; Nutrition, Physical Activity, and Metabolism Council; and the Quality of Care and Outcomes Research Interdisciplinary Working Group: The American Academy of Neurology affirms the value of this guideline. Stroke. 2006;37(6):1583-1633.

46. Blum A, Front E. Periodontitis, endothelial dysfunction and atherosclerosis. Vascular Disease Prevention. 2006;3(1):57-60.

47. Ameriso SF, Villamil AR, Barreto MP. Infection, inflammation, and stroke. Rev Esp Cardiol Supl. 2004;4 Suppl G:7-12.

48. Ricevuti G, Gritti D, Gasparetto C, et al. Immune activation as effect modifier of atherogenesis in chronic infection. Int Rev Immunol. 2002,21(1):27-31.

49. Grau A, Buggle F. Infection, atherosclerosis and acute ischemic cerebrovascular disease. Rev Neurol. 1999;29(9):847-51.

50. Corea F, Kwan J, Abbas MA. Predisposition to carotid atherosclerosis in ICARAS dental substudy. Stroke. 2007;38(1):12.

51. Sinzinger $\mathrm{H}$. Does the risk factor profile have predictive value for the site of atherosclerosis. J Neurol Neurosurg Psychiatry. 2005,76(8):1045.

52. Study links smoking and cardiovascular disease. Expert Review of cardiovascular therapy. 2005,3(2):191-2.

53. Söder B. Dental plaque as a possible risk factor for general disease - results from longitudinal studies. Int J Dent Hyg. 2006;4 (Suppl 1):22-5; discussion 50-2.

54. Mustapha IZ, Debrey S, Oladubu M, et al. Markers of systemic bacterial exposure in periodontal disease and cardiovascular disease risk: a systematic review and meta-analysis. J Periodontol. 2007;78(12):2289-302.

55. Aimetti M, Romano F, Nessi F. Microbiologic analysis of periodontal pockets and carotid atheromatous plaques in advanced chronic periodontitis patients. J Periodontol. 2007;78(9):1718-23.

56. Romano F, Barbui A, Aimetti M. Periodontal pathogens in periodontal pockets and in carotid atheromatous plaques. Minerva Stomatol. 2007;56(4):169-79.

57. Ford PJ, Gemmell E, Chan A, et al. Inflammation, heat shock proteins and periodontal pathogens in atherosclerosis: an immunohistologic study. Oral Microbiol Immunol. 2006;21(4):206-11.

58. Ford PJ, Gemmell E, Hamlet SM, et al. Cross-reactivity of GroEL antibodies with human heat shock protein 60 and quantification of pathogens in atherosclerosis. Oral Microbiol Immunol. 2005;20(5):296-302.

59. Fiehn NE, Larsen $T$, Christiansen $N$, et al. Identification of periodontal pathogens in atherosclerotic vessels. I Periodontol. 2005;76(5):731-6.

60. Glurich I, Grossi S, Albini B, et al. Systemic inflammation in cardiovascular and periodontal disease: comparative study. Clin Diagn Lab Immunol. 2002;9(2):425-32.

61. Chiu B. Multiple infections in carotid atherosclerotic plaques. Am Heart ). 1999;138(5 Pt 2):S534-6.
62. Haraszthy VI, Zambon JJ, Trevisan M, et al. Identification of periodontal pathogens in atheromatous plaques. J Periodontol. 2000,71(10):1554-60.

63. Brodala N, Merricks EP, Bellinger DA, et al. Porphyromonas gingivalis bacteremia induces coronary and aortic atherosclerosis in normocholesterolemic and hypercholesterolemic pigs. Arterioscler Thromb Vasc Biol. 2005; 25(7):1446-51.

64. Engebretson SP, Lamster IB, Elkind MSV, et al. Radiographic measures of chronic periodontitis and carotid artery plaque. Stroke. 2005;36(3):561-6.

65. Persson RE, Hollender LG, Powell VL, et al. Assessment of periodontal conditions and systemic disease in older subjects. II. Focus on cardiovascular diseases. J Clin Periodontol. 2002;29(9):803-10.

66. Desvarieux M, Demmer RT, Rundek T, et al. Periodontal microbiota and carotid intima-media thickness: the Oral Infections and Vascular Disease Epidemiology Study (INVEST). Circulation. 2005; 111(5):576-82.

67. Pussinen PJ, Nyyssönen K, Alfthan G, et al. Serum antibody levels to Actinobacillus actinomycetemcomitans predict the risk for coronary heart disease. Arterioscler Thromb Vasc Biol. 2005;25(4): 833-8.

68. Beck JD, Eke P, Lin D, et al. Associations between IgG antibody to oral organisms and carotid intima-medial thickness in community-dwelling adults. Atherosclerosis. 2005;183(2):342-8.

69. Taniguchi A, Nishimura Y, Nagasaka S, et al. Porphyromonas gingivalis infection is associated with carotid atherosclerosis in nonobese japanese type 2 diabetic patients. Metabolism. 2003;52(2): $142-5$.

70. Franek E, Blaschyk R, Kolonko A, et al. Chronic periodontitis in hemodialysis patients with chronic kidney disease is associated with elevated serum C-reactive protein concentration and greater intima-media thickness of the carotid artery. J Nephrol. 2006;19(3):346-51.

71. Genctoy G, Ozbek M, Avcu N, et al. Gingival health status in renal transplant recipients: relationship between systemic inflammation and atherosclerosis. Int J Clin Pract. 2007;61(4):577-82.

72. Vieira CLZ. Relação entre doenças periodontais e aterosclerose subclínica em indivíduos com hipercolesterolemia familiar. [tese]. São Paulo: Universidade de São Paulo, 2008.

73. Söder PO, Meurman JH, Jogestrand T, et al. Matrix metalloproteinase-9 and tissue inhibitor of matrix metalloproteinase- 1 in blood as markers for early atherosclerosis in subjects with chronic periodontitis. J Periodontal Res. 2009;44(4): 452-8.

74. Desvarieux M, Demmer RT, Rundek T, et al. Oral Infections and Vascular Disease Epidemiology Study (INVEST). Relationship between periodontal disease, tooth loss, and carotid artery plaque: the Oral Infections and Vascular Disease Epidemiology Study (INVEST). Stroke. 2003;34(9):2120-5.

75. Desvarieux M, Schwahn C, Völzke H, et al. Gender differences in the relationship between periodontal disease, tooth loss, and atherosclerosis. Stroke. 2004;35(9):2029-35.

76. Ravon NA, Hollender LG, Mc Donald V, et al. Signs of carotid calcification from dental panoramic radiographs are in agreement with Doppler sonography results. J Clin Periodontol. 2003;30(12): 1084-90. 
77. Bäck M, Airila-Månsson S, Jogestrand T, et al. Increased leukotriene concentrations in gingival crevicular fluid from subjects with periodontal disease and atherosclerosis. Atherosclerosis. 2007;193(2):389-94.

78. Demmer RT, Kocher T, Schwanhn C, et al. Refining exposure definitions for studies of periodontal disease and systemic disease associations. Community Dent Oral Epidemiol. 2008;36(6):493-502.

79. Söder B, Yakob M. Risk for the development of atherosclerosis in women with a high level of dental plaque and severe gingival inflammation. Int J Dent Hyg. 2007;5(3):133-8.

80. Cairo F, castellani S, Gori AM, et al. Severe periodontitis in young adults is associated with sub-clinical atherosclerosis. I Clin Periodontol. 2008;35(6):465-72.
Correspondência Rafaela das Mercês Batista Av. Resplendor, 563, sala 305 - Itapoã CEP 29101-500 - Vila Velha (ES), Brasil E-mail: rafinhambatista@hotmail.com

Contribuições dos autores Concepcão e desenho do estudo: RMB, AEO, ETS Análise e interpretação dos dados: RMB, EPR, EZ Coleta de dados: RMB Redação do artigo: RMB, EZ, EPR Revisão crítica do texto: RMB, AEO, EZ, EPR, MDCM, LH, ETS Aprovação final do artigo*: RMB, AEO, EZ, EPR, MDCM, LH, ETS Análise estatística: EZ Responsabilidade geral pelo estudo: RMB *Todos os autores leram e aprovaram a versão final submetida ao J Vasc Bras. 